\title{
Deep Learning techniques for energy forecasting and condition monitoring in the manufacturing sector
}

Victoria Jayne Mawson and Ben Richard Hughes

Department of Mechanical Engineering, The University of Strathclyde, Glasgow.

Keywords: Energy modelling, Manufacturing energy analysis, Energy Prediction, HVAC, Building energy analysis, Artificial Neural Networks, Recurrent Neural Networks

\begin{abstract}
The industrial and building sector demands the largest proportion of global energy, therefore adopting energy efficiency related strategies, optimization techniques and management is an important step towards global energy reduction. The use of machine learning techniques in energy forecasting is gaining popularity due to their ability to solve complex non-linear problems, however this is predominately seen in the residential and commercial sector. This study proposes and compares the use of two Deep Neural Networks, Feed Forward and Recurrent, to forecast manufacturing facility energy consumption and workshop conditions based on production schedules, climatic conditions, thermal properties of the facility building, along with building behaviour and use. The feed forward model was able to predict building energy, workshop air temperatures and humidity to an accuracy of $92.4,99.5$ and $64.8 \%$ respectively when the model was provided with a new dataset, with the recurrent model predicting these variables to accuracies of $96.82,99.40$ and $57.60 \%$. The neural networks were trained with data obtained from the simulation of a medium sized manufacturing facility in the UK. Coupling simulation techniques with machine learning algorithms allows for a low cost, non-intrusive methodology of predicting and optimising building energy consumption in the manufacturing sector. Furthermore, the use of neural networks provided forecasted building energy profiles for the identification of spikes in energy consumption; an undesirable and considerable cost in the manufacturing sector, as well as the predication of manufacturing environmental conditions for condition monitoring of condition sensitive production environments.
\end{abstract}

\section{Acronyms}

$\begin{array}{ll}\text { ANN } & \text { Artificial Neural Network } \\ \text { DES } & \text { Discrete Event Simulation } \\ \text { DFNN } & \text { Deep Feed Forward Neural Networks } \\ \text { DRNN } & \text { Deep Recurrent Neural Networks } \\ \text { HMM } & \text { Hidden Markov Models } \\ \text { HVAC } & \text { Heating, Ventilation and Air Conditioning } \\ \text { MAE } & \text { Mean Absolute Error } \\ \text { MLP } & \text { Multi-Layer Perceptron }\end{array}$




$\begin{array}{ll}\text { NAdam } & \text { Nesterov-Accelerated Adaptive Moment Estimation } \\ \text { ReLU } & \text { Rectified Linear Unit Function } \\ \text { RMSE } & \text { Root Mean Square Error } \\ \text { SVM's } & \text { Support Vector Machines }\end{array}$

\section{$\underline{\text { Nomenclature }}$}

$\mathrm{n} \quad$ number of samples in the training or testing set

$y_{i} \quad$ the actual output variable for entry $\mathrm{i}$

$\hat{y}_{i} \quad$ the predicted output variable.

\subsection{Introduction}

It is predicted that the worlds energy consumption will increase by over $50 \%$ before 2030 [1], with industrial energy use accounting for $37 \%$ of global energy use [2]. Adopting cost-effective energy efficiency opportunities, industry has the potential to double production value per unit of energy use in 2040 [2].

With increasing industrial demand along with rising energy prices, companies are required to adopt energy management strategies to allow for better utilisation of energy and a reduction in energy costs. For such strategies to be implemented, an understanding of both past and future energy demands and trends is required, along with an understanding of interactions between all building energy flows. This includes effects of occupant behaviour on energy demand, thermal comfort requirements, and energy flows from equipment within the facility. Along with high-powered energy consuming machinery, facilities have complex heating, ventilation and air conditioning (HVAC) systems to regulate workshop conditions.

HVAC systems are reactive systems, and aim to provide thermal comfort, playing a key role in ensuring certain levels of temperature and humidity, as well as the removal of $\mathrm{CO}_{2}$. The Manual of Recommended Practice states that although ventilation rates in industrial environments are based on rate of contaminant generation, of which is ensured by the HVAC system due to health risks, alongside this the primary function of heat control ventilation systems is to prevent the acute discomfort or illness of workers [3].

Such a system is ideal for office and domestic environments where thermal comfort of occupants is a priority, and less suited to manufacturing facilities, which may require production specific environmental conditions, of which are influenced by considerable heat and moisture gains from machinery. Humidity and temperature control, and therefore HVAC, can be vital for production quality control.

Furthermore, studies have shown that investing in energy-efficiency technologies and adopting technology to intelligently control energy use has the potential to reduce energy consumption by $50 \%$ as opposed to making operational improvements, of which can reduce this by only $10-20 \%$ [4]. However measures to reduce energy consumption are often limited by financial or regulatory constraints in large 
industrial facilities, and therefore there is an increased attention on energy management and system control.

Despite this, at present, many manufacturing facilities lack energy strategies [5], additionally over a third of manufacturing companies do not set energy efficiency targets nor have any means of measuring improvements [6]. Traditionally companies have been reluctant to modify the control or modelling of HVAC systems, and prefer the simplistic on/off based control [7]. Such control results in a high-energy consumption and high operating costs, as well as less than optimum indoor conditions. Furthermore, production managers do not generally account for energy costs, and are considered indirect costs to maintain facility operation [8].

Industrial environments are highly stochastic, with complex interactions between resources, water, compressed air, heat and energy at the system level, together with the manufacturing building, with the interaction of such manufacturing systems with that of the surrounding environment, influenced by facility design, solar radiation, HVAC operation and occupant behaviour. Energy systems in manufacturing facilities are complex, with facilities varying greatly in terms of building size, use, production scale and requirements. It is therefore difficult to pose a 'one fits all' solution to energy management.

The analysis of such complex systems is highly suited to simulation, with many studies adopting the use of Discrete Event Simulation (DES) for analysis of manufacturing systems, and time based simulation for building energy analysis. Without simulation, the size of data sets and relationships between variables becomes difficult to determine, and optimisation of such systems poses difficulty [8].

Furthermore, simulation is key to modelling and analysing the complex non-linear nature of HVAC systems due to the large number of subsystems (chillers, boilers, fans and pumps, heat exchangers and pipes). HVAC system demand is influenced by outdoor weather conditions, occupant behaviour, thermal gains from machining equipment, and therefore production schedules, as well as providing production specific environmental conditions.

Complex interactions between parameters in the built environment can be highly variable and can be difficult to understand and therefore model accurately. Furthermore, data from building energy systems and manufacturing systems is often noisy, making the problem highly suited to analysis with Artificial Neural Networks (ANN's).

There is an increase in the convergence of simulation models together with ANN's due to the increase in size of data sets, with the potential to improve data evaluation and analysis [9]. Furthermore, implementing sensors, setting up networks for data collection and training an ANN is time consuming, intrusive and expensive. Simulation can provide a tool to gather data in a lower risk manner, and provides the ability to group certain objects together for more effective data measurements and analysis, with the ability to improve ANN training speed. Learning from simulation is increasingly becoming a pre-requisite for projects and studies which require interactions with the real world [10].

In the field of energy analysis, ANN's are a popular forecasting tool due to their ability to solve non-linear problems with large data sets and little knowledge about the relationship between parameters. This study used simulation to understand the interactions between energy flows associated with the occupants, building shell, construction materials, machining schedules, building location and weather conditions and the requirements set by building regulations. Machine learning techniques, Deep Feed Forward Neural Networks (DFNN) and Deep Recurrent Neural Networks (DRNN), were trained from results on building 
behaviour from simulation, and used to predict facility energy demand based on weather conditions, machining schedules and building use. Furthermore, the networks were used to predict environmental conditions within the workshop to ensure quality control of condition sensitive production environments. Understanding relationships between energy flows from manufacturing systems and HVAC demand through the use of simulation can provide companies with additional insights and means of developing methods of reducing energy demand. Furthermore, such knowledge can aid in the understanding of energy and condition predictions from ANN's, and allow for well-informed strategies regarding high energy consumption predications to be made, such as shift pattern management or staggered starting of machining equipment.

\subsection{Previous Related Work}

ANN's followed closely by Support Vector Machines (SVM's) are considered the most widely used machine learning technique for energy prediction, with Hidden Markov Models (HMM) also gaining attention [11]. Hybrid approaches combining machine-learning techniques with statistical methods are also used to improve accuracy of certain models.

ANNs are a popular tool for energy demand forecasting and energy planning management due to their ability to approximate nonlinear processes to a high degree of accuracy. A review on energy models for electrical demand forecasting discusses the use of ANNs for studying short, medium and long term forecasting for electricity demand countrywide [12]. Social, economic and environmental factors are discussed as influencing factors to demand and required generating capacity of electricity.

The first found study using ANN's in the building sector for forecasting electrical energy was by Javeed and Al-Garni in 1995 [13]. The study used ANN's to forecast electrical energy consumption related to weather data and population. It was concluded that ANN's are an effective tool for energy forecasting and can be built with little data regarding the relationships between input variables.

Studies using ANNs in the commercial sector is a common theme, with Chae et al. [14] using a feed forward ANN to forecast electrical energy consumption of an office, gymnasium and cafeteria complex using outdoor air temperature, humidity, wind speed and building management system data. Likewise, Vázquez-canteli [15] used deep reinforcement learning for building energy management, computing cooling, heating and lighting energy demands at hourly intervals.

Mohammad and Kim [16] used a deep feed forward neural network for energy load forecasting in smart grids, and Gensler et al. [17] used LSTM for solar power forecasting, showing the potential of machine learning for energy demands on a larger scale.

Neto and Fiorelli [18] compared results from EnergyPlus simulations with an ANN model when forecasting building energy consumption paired with meteorological data for a university building. A model using external dry bulb temperature as the only input was tested before adding humidity and solar radiation as additional input parameters. The study concluded that the simulation model showed an error of $13 \%$ whereas the ANN model showed an error of $10 \%$ for a 7 -month period, however due to the large number of model uncertainties in evaluation of lighting, equipment and occupancy schedules, the study was considered preliminary and the methodology is to be developed further in order to provide more accurate forecasting. 
In the manufacturing sector, Cupek et al. presented a k-means clustering algorithm to monitor energy efficiency of compressed air systems [19]. K-means is an iterative unsupervised clustering algorithm which is used to find groups in a dataset, with data being split into one of $k$ groups. Energy consumption measurements and observations on machine behaviour allowed for the determination of energy profiles specific to different system states. These profiles were used to monitor energy efficiency and detect faults, as well as providing forecasts of energy consumption for given production requirements.

As well as the analysis of building level energy, studies have also focused on optimising sub-level components, such as HVAC systems.

Wang et al. [20] introduced a supervisory controlled HVAC system, of which sought the minimum energy input or operating cost to provide optimum indoor conditions, whilst accounting for changing indoor and outdoor conditions and characteristics of HVAC systems. However such a system is labour intensive, requiring operators input to apply rules to reduce the energy such as appropriate stop and start times, precooling and preheating. Furthermore, the system provided no insight into future conditions of the facility, and was unable to determine the best course of action for facility operation. Furthermore, a practical validation of such supervisory methods on real HVAC systems was missing, with validation only done on small-scale HVAC systems through the use of simulation. The authors introduced the use of ANN based control strategies for HVAC, suggesting the potential for energy or cost savings with their use.

Afram et al. [21] saw the disadvantage of the manual control based and non-predictive nature of such supervisory HVAC systems, and therefore used ANN models in a residential setting to determine temperature set-point profiles of zone air and buffer tank water, resulting in reduced operating costs of HVAC systems without compromising thermal comfort. However, it was stated that the requirement for training data covering all operating conditions could be challenging for large HVAC systems which operate over a wide range of weather conditions.

Petri et al. [22] coupled an ANN based optimisation module along with energy simulation in order to reduce energy consumption in a sporting facility whilst maintaining thermal comfort. The model was able to provide a method of reducing electrical and thermal energy consumption with additional control and uniformity over consumption intervals and balance between optimal objectives over a 42-day period. The ANN module was able to replace the simulation module in order to reduce computation time. However the study required constant monitoring and data collection with implementation of many sensors. A large number of input variables were also required, making data collection time consuming.

In the manufacturing sector, Azadeh et al. [23] used ANNs to predict long-term annual electricity consumption for high energy consuming manufacturing environments such as chemical, metal and mineral based industries. Such industries are prone to large fluctuations in energy consumption, and therefore analysis by regression models was unsuitable. The study showed that the use of ANNs can estimate annual consumption of manufacturing industries to a higher level of accuracy than statistical techniques, and shows the potential of such techniques.

Found studies focus predominantly on energy analysis of office and residential spaces, with little work done at energy predictions in industrial settings. 
Although ANNs are widely used in energy forecasting and electrical energy use prediction, DNNs with 2 hidden layers have been shown to produce the best results for monthly energy forecasting, peak load forecasting and daily total load forecasting [24].

Berriel et al. [25] suggested that deep neural networks hold the most potential for energy consumption forecasting, and present a system for energy companies to forecast monthly energy consumption at the customer level. Three neural network techniques were evaluated, Deep Fully Connected, Convolutional and Long Short Term Memory (Recurrent). The system was based on a temporal sequence of energy consumption alongside customer attributes such as customer class (residential, business, industrial etc). Training was based on past 12 months of energy consumption data for each customer. However the methodology required monthly data collection and energy meter readings from all customers; a time consuming and error prone method of data collection. The study concluded that the system outperformed the energy forecasting methodology currently used by the discussed power company.

Amasyali and El-Gohary stated that deep learning based models outperform other machine learning algorithms in many field including building energy predication [22]. The study presented a deep learning based model to predict hourly cooling energy consumption of an office building based on outdoor weather conditions, and compares these results against other machine learning models and statistical regression based methods. The models were trained with simulation-generated data, and predictions made by the DNN model compared to that of the simulation model, and was considered to have achieved good accuracy and computational efficiency.

No studies were found using deep learning for the optimisation of HVAC systems, nor HVAC systems in the manufacturing sector. Furthermore, there were no found studies on energy prediction or environmental condition based prediction using DNNs in the manufacturing sector.

The research gap was identified as a lack of research on the application of deep learning techniques to the manufacturing sector. The novelty in this study arises from the use of these techniques to predict not only building energy but also indoor conditions of which is important to monitor and control for thermal comfort and production quality control. The use of simulation coupled with machine learning allows for scenario testing and optimisation based on predicted conditions and building energy, for further energy savings and knowledge gain on thermal flows in the production environment. This study aimed to develop a deep learning approach to building energy forecasting in the manufacturing sector for the identification of spikes in energy consumption and determined the interaction between manufacturing demand and facility energy consumption. Furthermore, the prediction of indoor facility conditions allowed for condition monitoring of which is essential in condition sensitive production environments. Identifying spikes in energy consumption along with undesirable workshop conditions allows for HVAC control optimisation and efficient scheduling of production.

\subsection{Methodology}

In this study, a simulation model of a manufacturing environment was created to determine thermal energy flows in and around the building, accounting for influencing factors such as climate, occupancy, 
building fabrics, HVAC control, internal gains, manufacturing schedules, building regulations and airflows. This simulation was used to determine building behaviour, of which could be used to train the DFNN and DRNN, which ultimately provided predictions for indoor humidity and temperature as well as building energy consumption.

\section{$\underline{2.1}$ Simulation model}

In order to obtain training data for the DNNs in this study, a dynamic simulation of a research focused manufacturing facility was run using a case study of a medium sized two floor (6400 sq.m) manufacturing facility. The model was split into 59 thermal zones corresponding to different rooms within the building, with the manufacturing workshop being on the first floor only. Simulation tools used for Building Energy Modelling are usually time driven, where a simulation variable is incremented at set time intervals with computation at each interval, which is well suited to simulation of thermal energy flows, airflows and complex fluid dynamics. Components within a building such as equipment, ovens and computers could be modelled alongside occupant behaviour and climatic conditions, whilst ensuring the building runs to regulatory standards. Many software packages have been developed for building energy analysis, such as ESP-r [26], Energy-Plus [27] and DOE-2 [28]. In this study, IES-VE, dynamic time based building energy simulation software, was utilised as the simulation tool. As well as modelling building performance, the tool also allows construction of manufacturing components, allowing the user to model manufacturing heat sources within a space, and user defined objects specified by heat gains or surface temperature. Manufacturing schedules could also be specified, along with a corresponding heat gain profile specifying the amount of heat released from the equipment at certain points during the day.

Data to build the simulation was obtained through interviews with machine operators and management, through machine booking systems and from manufacturing data booklets. Discussions with facility staff combined with electricity consumption history and production schedules provided insight into the relationship between energy consumption of machines and building on working and non-working days. Building fabrics, construction material and building height information was obtained based on observation and discussion with managers, and also based upon common construction techniques and methods at the time of build, 2008. The HVAC system was a Schneider PM200 system, set to operate 24 hours a day, based on the thermal comfort of occupants. The site is part of a large industrial complex, of which is supplied with a $11 \mathrm{kV}$ electrical supply. Weather data was provided as part of the IES-VE ApLocate weather and location editor.

\subsubsection{Model Validation}

Energy data from the building obtained from pre-installed energy data collection systems at the manufacturing facility was used to validate the IES-VE model. Meters in the building only collect energy data on a building level, and therefore sub-metered data for individual components such as energy consumption of lighting and HVAC systems was not available.

The IES-VE model with and without manufacturing equipment was compared with the metered energy data for a simulation period of 1 year (Table 1). It should be noted that only variables influenced by manufacturing processes are displayed in the table. Energy from additional sources such as lighting and computers is included in the table; however, such factors contributed towards the final energy requirement of the facility. 
Table 1- Building Energy Analysis- IES-VE and Metered data

\begin{tabular}{|l|l|l|l|}
\hline & $\begin{array}{l}\text { With manufacturing } \\
\text { equipment } \\
(\mathrm{MWh})\end{array}$ & $\begin{array}{l}\text { Without manufacturing } \\
\text { equipment } \\
(\mathrm{MWh})\end{array}$ & Metered data \\
$(\mathrm{MWh})$
\end{tabular}

The IES-VE model underestimated total energy consumption for a simulation period of 1 year by $6 \%$ in comparison to metered data. These errors are likely to arise due to the unavailability of data to build the simulation such as accurate details regarding building construction materials, occupancy patterns, lack of data regarding machining times and length of certain machining jobs, lighting data and also lack of knowledge on occupant behaviour and location. For example, the IES-VE model was provided with data on a set number of occupants in each room dependant on its use, however, in reality, stochastic and unpredictable occupant behaviour can have a significant impact on building energy consumption, of which is unquantifiable in simulation without real detailed metered data within the model. From simulation, it was concluded that manufacturing processes accounted for $19 \%$ of the total energy use of the facility comparing an environment with equipment to one without.

\subsection{Artificial Neural Network}

\subsubsection{Structure of ANN- number of layers}

A neuron in a neural network is a unit which has weighted input connections, a transfer function to combine the inputs and output. These neurons are organised into layers which builds up the neural networks structure. A single layer structure is used for simple linear functions, however more complex relationships and non-linear relationships require multi-layer networks; Multi-Layer Perceptrons (MLPs).

A number of methods can be used to determine the optimum number of layers within the network, as well as the number of neurons within these layers. These involve experimentation and trial and error until a suitable architecture is found, however this can be time consuming for large structures. Systematic grid search, where a search across the number of layers and nodes per layer is performed, was used in this study in order to find the optimum network, which consisted of two hidden layers. 


\subsubsection{Pre-processing of Data}

Training data regarding the building was provided from IES-VE simulations, whereas data regarding machine use and schedules was provided from machine schedule databases. Inputs to the DNN included weather conditions such as outdoor dry bulb temperature, outdoor wind speed, cloud cover and outdoor relative humidity, as well as machining schedules. Weather conditions were sourced from Met Office databases, whereas machine schedules were obtained from facility databases and machine booking systems. Outputs from the DNN included relative humidity and temperature in the workshop and also building energy consumption.

A total of 29,568 sample data points were collected, with data from both cooler and warm seasons collected in order to gain information on building and HVAC behaviour in a range of climatic conditions.

As a DNN maps inputs to outputs, the scale of inputs and outputs used to train the model is crucial to providing a good model. Such data normalisation and standardisation techniques can ensure model convergence, and reduce the risk of exploding gradients and slow learning processes [29].

The aim of data standardisation and scaling is to ensure features more or less represent normally distributed data. This can be ensuring features are in the range $[0,1]$ or a standard normal distribution with zero mean and unit variance.

In this study, the data, $x$, was scaled to a range of $[0,1]$ (Equation 1).

$$
x_{\text {norm }}^{(i)}=\frac{x^{(i)}-x_{\min }}{x_{\max }-x_{\min }}
$$

Equation 1- Min-max scaling

Missing values in a dataset can also lead to unpredictable results and therefore these were removed from the dataset prior to data processing. Furthermore, as the facility undergoes complete shutdown overnight, non-occupied hours were removed from the dataset as these hours had different operational characteristics and specific workshop conditions is not required during this time. Such a methodology was also adopted by Platon et al. [30] for hourly prediction of a buildings electricity consumption and by Amasyall et al. [31] who took a deep learning approach to building energy consumption prediction.

\subsubsection{Training the network}

The activation function connects the weights of a neuron to the input, $x$, and determines the activation or the state of the neuron. It keeps values moving forward to subsequent layers within an acceptable and useful range, and towards the output, $y$.

The rectified linear unit function (ReLU) was used as the activation function in the hidden layers of the DNN.

$$
y=\max (0, x)
$$

Equation 2- ReLU activation function

ReLU is a commonly used activation function for DNN, and is less computationally expensive than other alternatives. Furthermore, it doesn't activate all neurons at once making computation quicker and easier. 
Initially, the weights of the network are assigned random values, so the network implements a series of random transformations. However, the resultant predications obtain with high errors. As the network runs through a training loop over every example fed to the network, the weights are updated a little in the correct direction, and the error decreases. With sufficient iterations provides weight values that minimize the loss function.

An optimization algorithm is used to minimize this function, which quantifies how wrong the prediction is. The most popular optimisation algorithm is gradient descent, which calculates what a change to each weight would do to the cost function, and adjusts each weight based on its gradient, until the loss function gets as low as possible.

In this study, the Nesterov-accelerated Adaptive Moment Estimation (NAdam) optimiser was used, an adaptive learning rate optimisation algorithm developed for use in DNNs. The optimiser was chosen from five other algorithms using a grid search iterative technique.

Momentum can be used to accelerate gradient descent learning by smoothing out oscillations and accelerating gradient decent where the gradient remains relatively consistent across training steps [32]. Nesterov momentum is thought of as an improved momentum, where momentum is applied before computing gradients for a 'superior step direction', and the use of NAdam has shown dramatic improvement in algorithms over the use of Adam optimisers [32], [33].

\subsubsection{Cross Validation}

$\mathrm{K}$ fold cross validation algorithm was used for validation, which shuffles and splits the dataset into $\mathrm{k}$ groups/ folds, where the first set is held as a validation set, and the rest used to train the model. This process is repeated until every $k$ fold has been used as the test set. The value of $k$ is commonly 5 or 10 [34], with 10 being used in this study. This resampling method was chosen to avoid bias caused by randomness in choosing testing and training sets [35], with every observation in the original dataset having a chance at being selected in training and test data.

\subsubsection{Accuracy Techniques}

Prediction accuracy was determined by using the mean absolute error (MAE), Equation 3, and root mean square error (RMSE), Equation 4, evaluation metrics, which are the mostly utilised performance metrics for ANNs [36].

$$
M A E=\frac{1}{n} \sum_{i=1}^{n}\left|y_{i}-\hat{y}_{i}\right|
$$

Equation 3- Mean absolute error

$$
R M S E=\sqrt{\frac{1}{n} \sum_{i=1}^{n}\left(y_{i}-\hat{y}_{i}\right)^{2}}
$$


Where $\mathrm{n}$ is the number of samples in the training or testing set, $y_{i}$ is the actual output variable for entry $\mathrm{i}$, and $\hat{y}_{i}$ is the predicted output variable.

The MAE measures accuracy and the average magnitude of errors in a prediction set, and is commonly used as performance metrics for energy forecasting with the use of ANNs [21], [25], [36]-[39]. The RMSE measures the average magnitude of the error, seen in a number of energy forecasting studies [12], [30], [36], [39], [40]. However RMSE gives high weight to large errors and therefore is useful when large errors should be avoided. Both metrics were used to compare models with one another throughout the optimisation process. For the DFNN, RMSE for the final model was 0.097, with MAE resulting to 0.060. For the DRNN, RMSE for the final model was 0.068 , with MAE resulting to 0.040 .

The final model was a multi-output neural network, and therefore Cv(RMSE) and MBE statistical analysis were not used as a performance metric.

\subsection{Use of Degree Days for Energy Analysis}

The use of degree days is commonly used to determine the effect of weather conditions on energy demand to plan energy systems, prediction seasonal load demands and to determine weather related variations in energy demand. Such a method is considered an index of the energy consumption of buildings and represents a method of estimating heating and cooling requirements of HVAC systems [41]. The CIBSE guide recommends a base temperature of $15.5^{\circ} \mathrm{C}$ [42] for calculation of degree days, however, this method is suitable for environments with consistent operational profiles and use, such as schools and office blocks. Manufacturing facilities however have rapidly fluctuating energy demands dependant on use, occupant behaviour and internal gains, especially heat gains from manufacturing equipment, which questions the applicability of the degree day method to manufacturing facilities. To determine the extent to which manufacturing schedules and heat gains are required during the analysis of building and HVAC energy as opposed to the degree day method, statistical tests were run, with results discussed in Section 3. Furthermore, the DFNN was run a second time, utilising degree days as opposed to external outdoor temperature to determine impact on energy predictions.

\subsection{Results and Discussion}

The final DFNN and DRNN from section 2.2 were used to predict workshop air temperature, humidity and total facility energy consumption at 1-hour intervals based on a set of unseen data obtained from the simulation model.

The final model DFNN was able to predict workshop air temperature to an accuracy of $99.5 \%$, with similar results seen using the DRNN model, with predictions to an accuracy of $99.4 \%$. As air temperature within the facility did not fluctuate significantly, with any fluctuations seen incrementally, the feedback loop within the recurrent model was seen to hold no advantage to the accuracy of the prediction. The data used to train the DFNN was sufficient to make accurate predictions based on machining schedule and building use.

The DFNN predicted workshop humidity to an accuracy of $64.8 \%$, with the DRNN model predicting to an accuracy of $57.6 \%$. The models predicted relative humidity to a relatively high accuracy in the morning shift of work, however during the last 1-2 hours of the working day, significant errors were obtained which led to the significant error in humidity. Relative humidity is highly impacted by occupancy, as well 
as $\mathrm{CO} 2$ concentration- due to occupants and waste gases from machining equipment. Therefore, a second model was run with the inclusion of workshop $\mathrm{CO} 2$ concentration as an input, which increased the accuracy of humidity prediction to $77.5 \%$ using the DFNN model and $78.6 \%$ using the DRNN model. However, there was no data available on occupancy for use as further training data. It was concluded that further inputs regarding occupant numbers was required in order to improve the accuracy of humidity predictions. Data collected for humidity within the workshop fluctuated significantly, with fluctuation trends based on machining schedules, and therefore also occupant numbers due to machining shifts, as opposed to time specific fluctuations. Here we can question the advantage of the recurrent model for such a prediction.

The DFNN predicted facility energy consumption to an accuracy of $92.4 \%$, with the recurrent model predicting energy consumption to an accuracy of $96.8 \%$.

Figure 1 displays predicted energy consumption from the DFNN in comparison to the observed energy consumption based on the simulation model, in hourly time steps. The DFNN under predicted energy consumption in most instances.

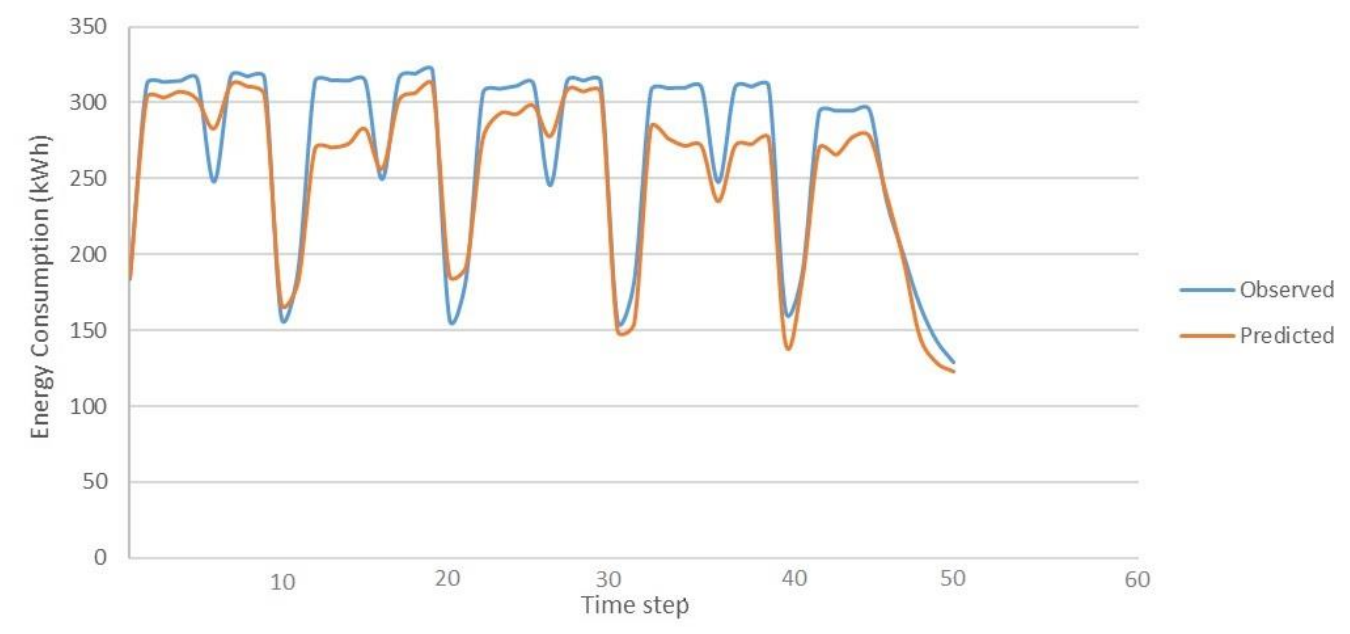

Figure 1- Predicted vs observed energy consumption for the DFNN model

Likewise, Figure 2 displays predicted energy comsumption from the DFNN in comparison to observed energy consumption based on the simualtion model, in hourly time steps. In comparison, the predictions made by the DRNN are less prone to underprediction than the DFNN. 


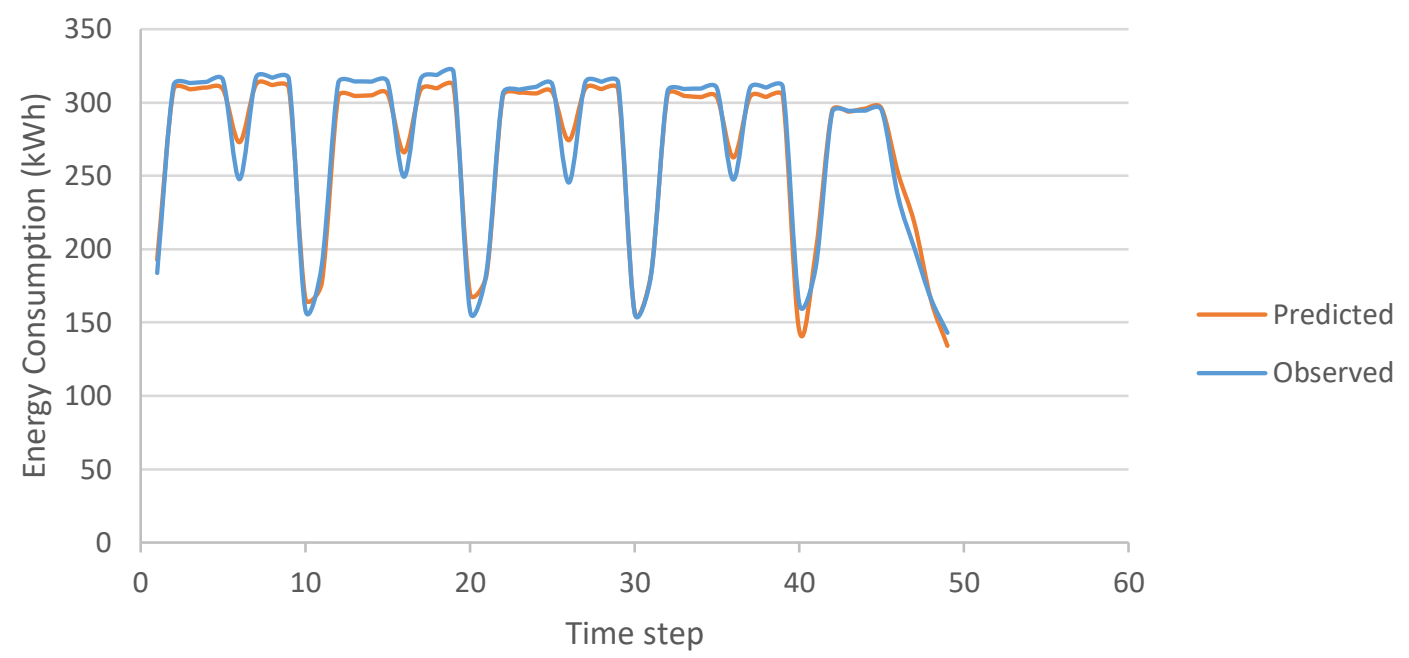

Figure 2- Predicted vs observed energy consumption for the DRNN model

Running simulation studies prior to predictions using DNN can also disclose important information regarding building behaviour, and intereations between machining schedules and HVAC energy consumption. Figure 3 displays machining demand against energy consumption from the HVACs chiller and boiler systems for a week in August. The chiller energy consumption profile coincides with the machining demand profile, with the system working to combat heat gains from manufaturing equipment and other internal gains such as occupants and computers. Boiler energy displays a peak early in the morning, prior to workers arriving and start of machining schedules. This quickly reduces as machining begins and workshop temperatures rise. 


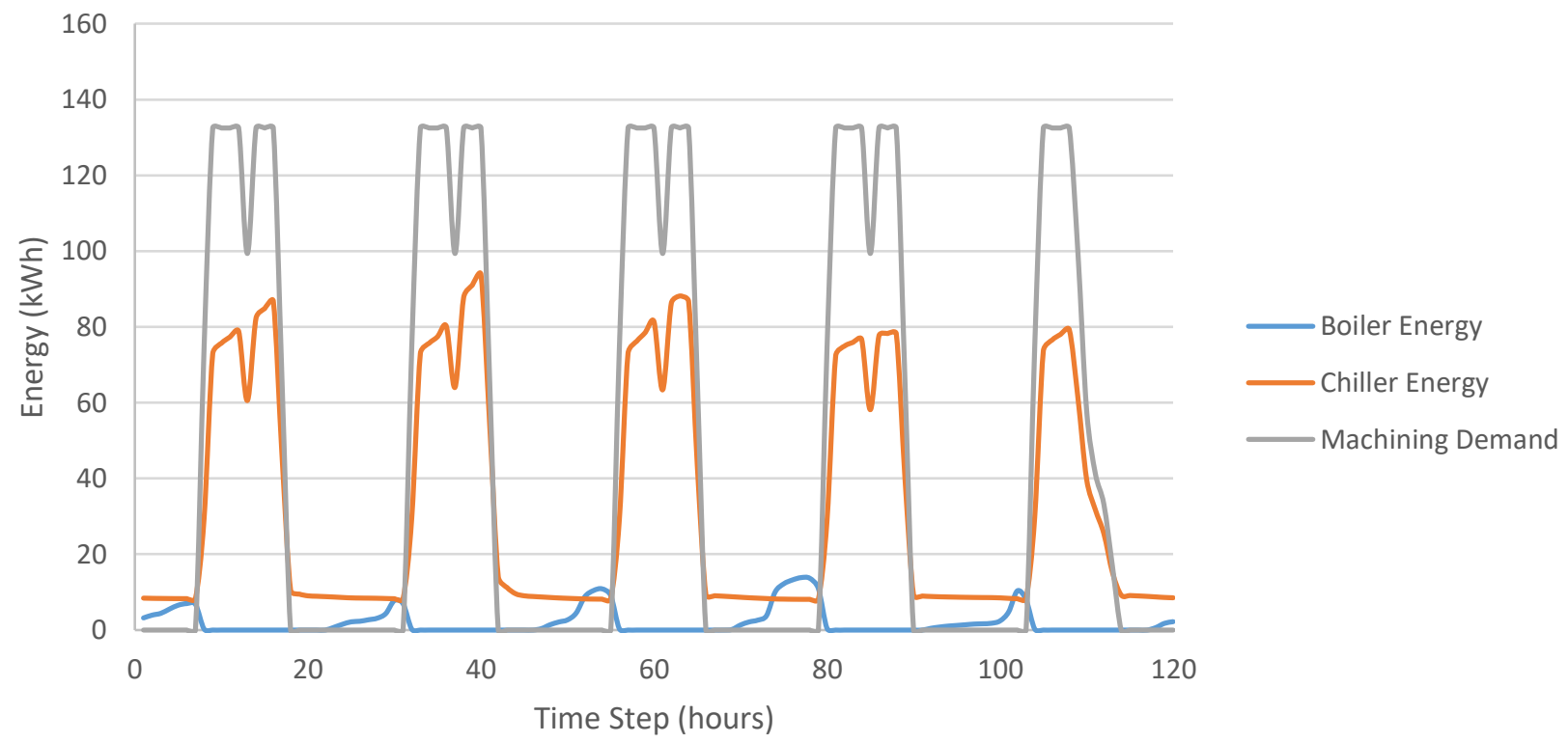

Figure 3- System energy alongside machining demand for a week in August

A similar trend is seen for the facility for a week in February, however during workshop shut down, due to the cool outdoor temperatures, the boiler systems are on constantly during the night and times of no machining, in order to ensure the facility does not plummet to extreme temperatures (Figure 4). 


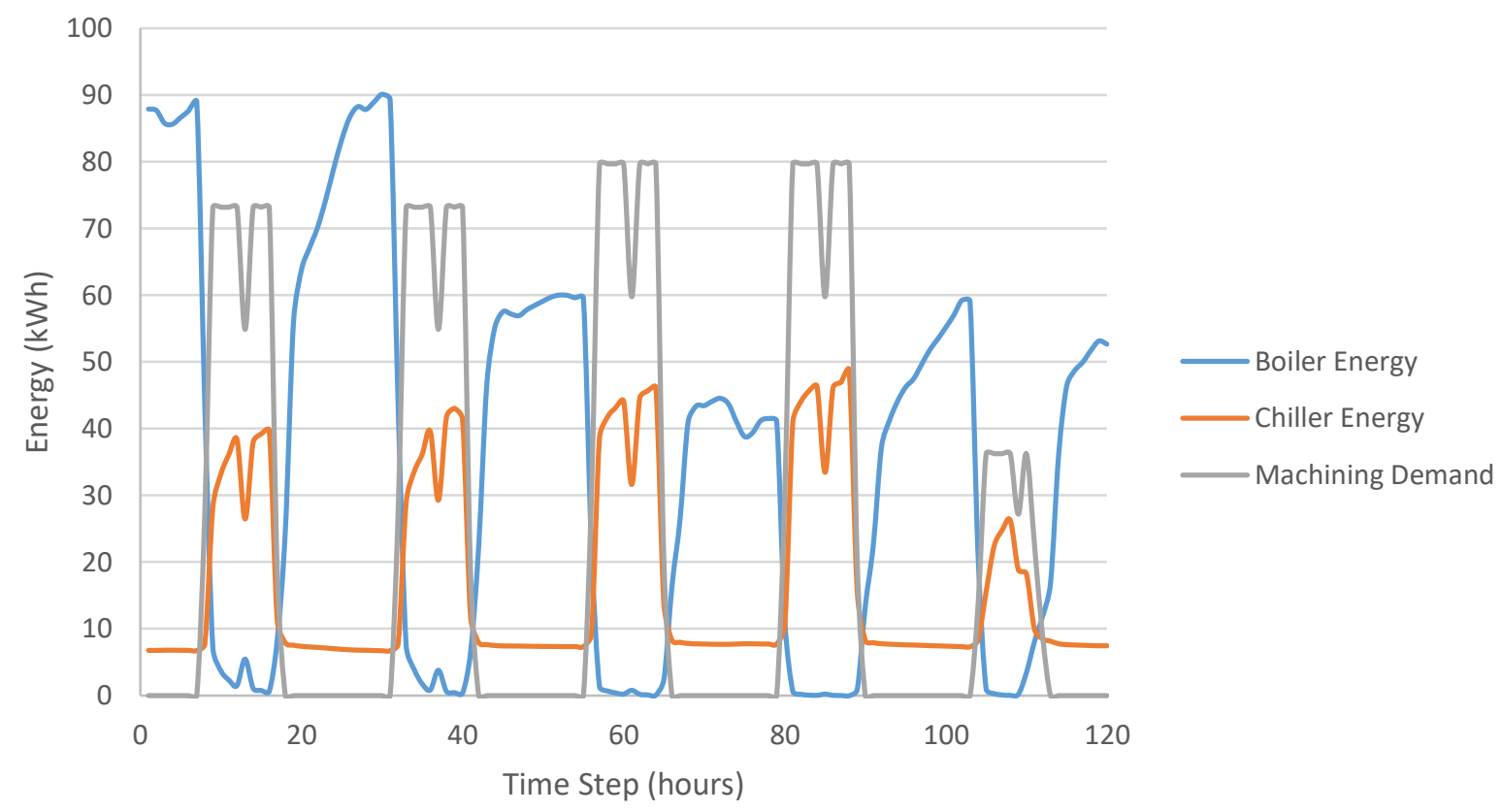

Figure 4- System energy alongside machining demand for a week in February

Being able to understand this interaction can allow for a greater understanding of energy consumption patterns, and displays the dependance of HVAC energy consumption and control on manufacturing schedules, and therefore allow energy strategies to implemented which are specific to the facility and its schedule.

Industrial facilities are charged for the greatest amount of energy they use over a 15-minute interval within a billing period, regardless of the total energy consumption, therefore such large fluctuations in the energy consumption of the facility can have a considerable effect on the price in which the facility is charged for its energy consumption. This spike in energy consumption can make up to $30 \%$ of the monthly operating cost of a manufacturing facility [43]. Energy visualisation techniques were adopted to correlate energy use with manufacturing production output to provide a greater understanding and context of energy use. Figure 5 displays predicted building energy consumption correlated with machining production for the DFNN model. 


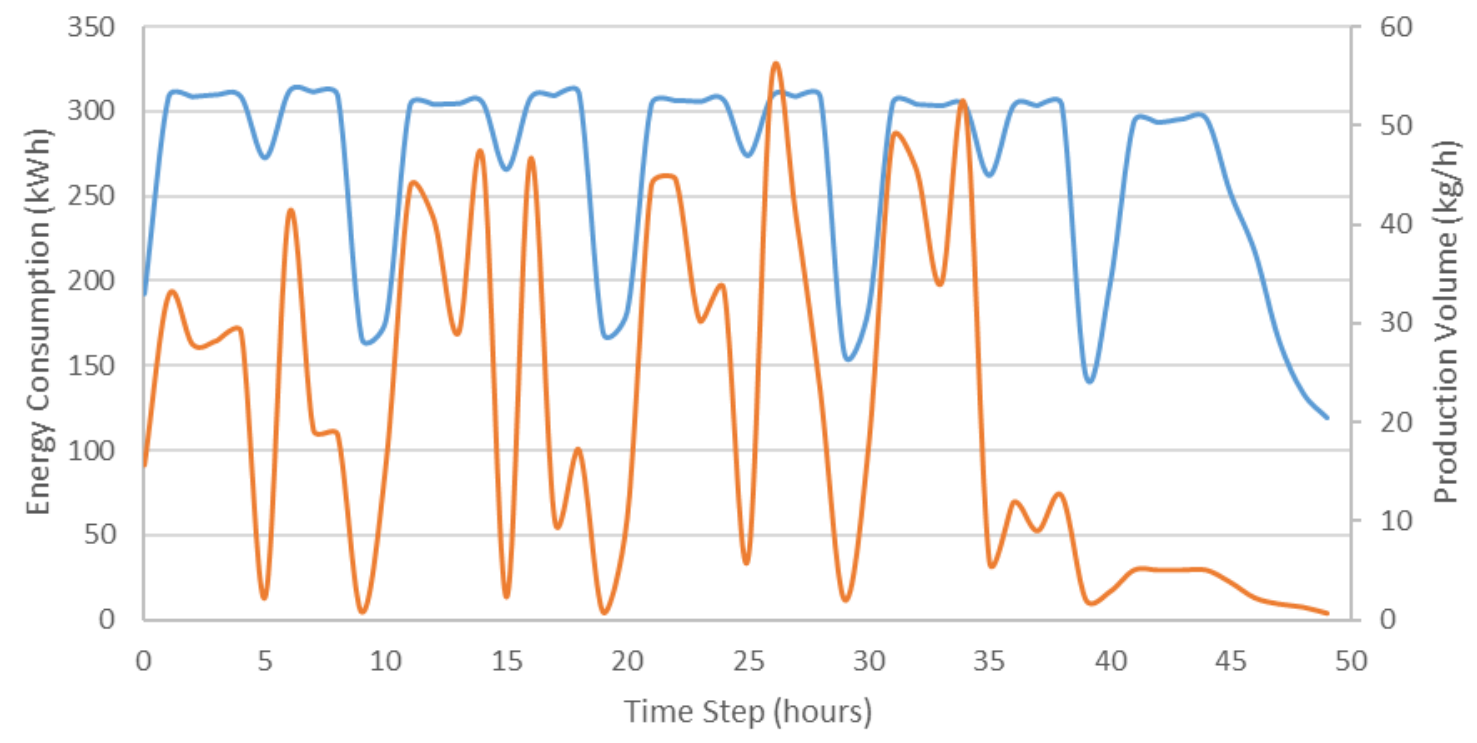

- Energy Consumption — Production Volume

Figure 5- Predicted facility energy consumption vs machining production for DFNN model

A spike in machining production and energy consumption can be seen between the $25^{\text {th }}$ and $30^{\text {th }}$ time step, with a dramatic dip after. With this prior knowledge of the interaction between machining demand and HVAC energy, as well as building behaviour and prediction of energy spikes, steps can be taken towards machining schedule optimisation to provide a less oscillatory energy profile, such as shift pattern management. Intense machining could be planned for earlier in the morning or later in the afternoon on warmer days to avoid coinciding intense machining, and thus a significant amount of waste heat, with peak mid-day temperatures in order to reduce demand on HVAC systems. Furthermore, adopting different shifts for workers can ensure that not all workers break at the same time, and worker schedules could adopt a staggered approach, to avoid a spike in consumption when all machines are turned on at the same time.

The degree day DNN model predicted building energy consumption with an accuracy of $88.3 \%$, a slight reduction in accuracy compared to the model based on outdoor temperatures. To determine the suitability of degree days for the analysis of energy consumption of manufacturing facilities as well as the energy consumption of HVAC systems, the correlation coefficient was determined between building and HVAC energy consumption with degree days, as well as the correlation between building and HVAC energy consumption with waste heat from machines/ machining schedules.

The results are displayed in Figure 6, where a value of 0 corresponds to zero correlation, and a value of 1 corresponds to positive correlation. 


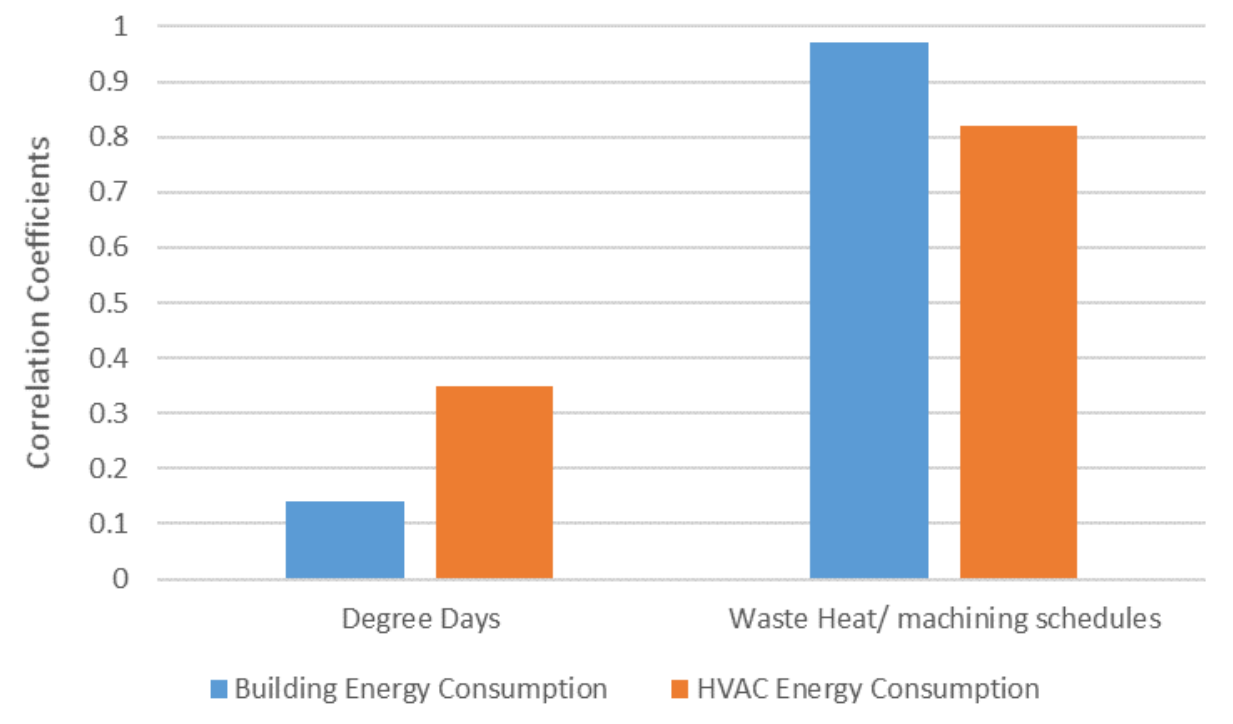

Figure 6- Correlation coefficient between energy consumption and degree days

and machining schedules

The correlation between energy consumption and degree days for the analysed manufacturing facility was weak, however the correlation between machining waste heat/ machine schedules was substantial, questioning the suitability of the degree day method for energy analysis of manufacturing facilities. Manufacturing equipment is a considerable consumer of energy in manufacturing environments, with equipment greatly influencing indoor conditions due to the release of heat and moisture during operation. Therefore, information regarding schedule and manufacturing demand is essential for accurate energy analysis of the facility and HVAC system, rather than being solely reliant on the degree day method.

\subsection{Conclusion}

Two deep learning based neural network models were presented for hourly energy and workshop environmental condition forecasting. Results show that the presented models were able to predict energy consumption and workshop air temperature to a high accuracy, and confirms the suitability and potential of Deep Learning models for energy prediction. The recurrent neural network model outperformed the feed forward neural network model for predictions of building energy consumption, with similar results seen in the prediction of indoor air temperature for both models. The feed forward model slightly outperformed the recurrent model for humidity predictions, however more work is required at improving this accuracy for both models. Such results paired with visualisation tools can be used to identify future spikes in energy consumption, of which is essential in avoiding larger tariffs for energy that is not necessarily used, of which can make up $30 \%$ of the monthly utility cost [43].

The predication of spikes in energy consumption can be correlated with machining schedule, and therefore machining energy, as well as HVAC energy consumption to provide opportunities for schedule optimisation. For example, avoiding intense machining at mid-day when outdoor temperatures increase, resulting in reduced demand on HVAC cooler and ventilation systems, and ensuring the environment does 
not reach temperatures and humidity levels that the HVAC system cannot compensate for, effecting production conditions and quality.

Furthermore, forecasting air temperatures and humidity in the workshop ensures that HVAC systems have the capability to compensate for any heat and moisture gains from machining equipment, and ensures optimum conditions for quality control.

The use of simulation can disclose interactions between manufacturing schedules with the energy consumption and demands of the HVAC system, and when coupled with prediction techniques, managers have a greater understanding regarding reasons for changes in environmental conditions and spikes in energy consumption. Thus, making more informed decisions regarding HVAC optimisation and energy management of the facility.

The inclusion of manufacturing heat gains and schedules during the energy analysis of manufacturing facilities is concluded, with the suitability of the degree day method for manufacturing environments questioned.

A high error was obtained for prediction of indoor humidity. Further work is required in gathering data regarding occupants through the use of methods such as occupancy sensors and passive infrared sensors. Such data would provide additional data inputs to the DNN, and provide a more detailed model of the buildings behaviour.

\section{Acknowledgements}

The authors would like to acknowledge the financial contribution provided by the Engineering and Physical Sciences Research Council (EPSRC) and the University of Strathclyde that has enabled the production of this paper.

\section{$\underline{\text { References }}$}

[1] A. The Natural Edge Project, CSIRO, and Griffith University, Energy Transformed: Sustainable Energy Solutions for Climate Change Mitigation, 2007.

[2] I. E. Agency, "Energy Efficiency: Industry," 2019. [Online]. Available: https://www.iea.org/topics/energyefficiency/industry/. [Accessed: 21-Jun-2019].

[3] HM Government, "Non-Domestic Building Services Compliance Guide 2013 edition - for use in England," NBS, part RIBA Enterp. Ltd, 2013.

[4] M. Company, "Technologies that could transform how companies use energy," 2015.

[5] A. Fysikopoulos and G. Pastras, "An Approach to Increase Energy Efficiency Energy Efficiency at Production Line Level," Adv. Prod. Manag. Syst. Innov. Knowledge-Based Prod. Manag. a Glob. World SE - 6, vol. 439, pp. 205-212, 2014.

[6] T. Manufacturer, "Failure to prioritise resource efficiency," 2018. . 
[7] A. Afram, F. Janabi-Sharifi, A. S. Fung, and K. Raahemifar, "Artificial neural network (ANN) based model predictive control (MPC) and optimization of HVAC systems: A state of the art review and case study of a residential HVAC system," Energy Build., vol. 141, pp. 96-113, 2017.

[8] G. S. Rodrigues, J. C. Espíndola Ferreira, and C. R. Rocha, "A novel method for analysis and optimization of electric energy consumption in manufacturing processes," Procedia Manuf., vol. 17, pp. 1073-1081, 2018.

[9] A. Tolk, "The Next Generation of Modeling \& Simulation : Integrating Big Data and Deep Learning," Summer Simul. Conf., no. June, pp. 1-8, 2015.

[10] S. Ruder, "Transfer Learning," 2017. [Online]. Available: http://ruder.io/transfer-learning/. [Accessed: 03-May-2018].

[11] E. Mocanu, P. H. Nguyen, M. Gibescu, and W. L. Kling, "Comparison of machine learning methods for estimating energy consumption in buildings," in 2014 International Conference on Probabilistic Methods Applied to Power Systems (PMAPS), 2014.

[12] L. Suganthi and A. A. Samuel, "Energy models for demand forecasting - A review," Renew. Sustain. Energy Rev., vol. 16, no. 2, pp. 1223-1240, 2012.

[13] N. S. Javeed and A. Z. Al-Garni., "Forecasting electric energy consumption using neural networks.," Energy Policy, vol. 23, no. 12, pp. 1097-1104, 1995.

[14] Y. T. Chae, R. Horesh, Y. Hwang, and Y. M. Lee, "Artificial neural network model for forecasting sub-hourly electricity usage in commercial buildings," Energy Build., vol. 111, pp. 184-194, 2016.

[15] J. R. Vázquez-Canteli, S. Ulyanin, J. Kämpf, and Z. Nagy, "Fusing TensorFlow with building energy simulation for intelligent energy management in smart cities," Sustain. Cities Soc., vol. 45, no. November 2018, pp. 243-257, 2019.

[16] F. Mohammad and Y.-C. Kim, "Energy load forecasting model based on deep neural networks for smart grids," Int. J. Syst. Assur. Eng. Manag., 2019.

[17] A. Gensler, J. Henze, B. Sick, and N. Raabe, "Deep Learning for solar power forecasting - An approach using AutoEncoder and LSTM Neural Networks," 2016 IEEE Int. Conf. Syst. Man, Cybern. SMC 2016 - Conf. Proc., no. April, pp. 2858-2865, 2017.

[18] A. H. Neto and F. A. S. Fiorelli, "Comparison between detailed model simulation and artificial neural network for forecasting building energy consumption," Energy Build., vol. 40, no. 12, pp. 2169-2176, 2008.

[19] R. Cupek, A. Ziebinski, D. Zonenberg, and M. Drewniak, "Determination of the machine energy consumption profiles in the mass-customised manufacturing," Int. J. Comput. Integr. Manuf., vol. 00, no. 00, pp. 1-25, 2017.

[20] S. Wang, Z. Ma, and S. Wang, "Supervisory and Optimal Control of Building HVAC Systems : A Review Supervisory and Optimal Control of Building HVAC Systems : A Review," HVAC\&R Res., vol. 9669, no. August 2015, pp. 37-41, 2011.

[21] A. Afram, F. Janabi-Sharifi, A. S. Fung, and K. Raahemifar, "Artificial neural network (ANN) based model predictive control (MPC) and optimization of HVAC systems: A state of the art review and case study of a residential HVAC system," Energy Build., vol. 141, pp. 96-113, 2017. 
[22] I. Petri, H. Li, Y. Rezgui, C. Yang, B. Yuce, and B. Jayan, "A modular optimisation model for reducing energy consumption in large scale building facilities," Renew. Sustain. Energy Rev., vol. 38, pp. 990-1002, 2014.

[23] M. A. Azadeh and S. Sohrabkhani, "Annual electricity consumption forecasting with Neural Network in high energy consuming industrial sectors of Iran," Proc. IEEE Int. Conf. Ind. Technol., vol. 49, pp. 2166-2171, 2006.

[24] T. Yalcinoz and U. Eminoglu, "Short term and medium term power distribution load forecasting by neural networks," Energy Convers. Manag., vol. 46, no. 9-10, pp. 1393-405, 2005.

[25] R. F. Berriel, A. T. Lopes, A. Rodrigues, F. M. Varejao, and T. Oliveira-Santos, "Monthly energy consumption forecast: A deep learning approach," Proc. Int. Jt. Conf. Neural Networks, vol. 2017May, pp. 4283-4290, 2017.

[26] U. of Strathclyde, "ESP-r," 2018. [Online]. Available: http://www.esru.strath.ac.uk/Programs/ESPr.htm. [Accessed: 13-Nov-2018].

[27] EnergyPlus, "EnergyPlus," 2018. [Online]. Available: https://energyplus.net/. [Accessed: 13-Nov2018].

[28] DOE-2, “DOE-2,” 2019. [Online]. Available: http://doe2.com/. [Accessed: 27-Jun-2019].

[29] J. Brownlee, "How to Improve Neural Network Stability and Modeling Performance with Data Scaling," 2019. [Online]. Available: https://machinelearningmastery.com/how-to-improve-neuralnetwork-stability-and-modeling-performance-with-data-scaling/. [Accessed: 24-Jun-2019].

[30] R. Platon, V. R. Dehkordi, and J. Martel, "Hourly prediction of a building's electricity consumption using case-based reasoning, artificial neural networks and principal component analysis," Energy Build., vol. 92, pp. 10-18, 2015.

[31] K. Amasyali and N. El-Gohary, "Deep Learning for Building Energy Consumption Prediction," Leadersh. Sustain. Infrastructure, CSCE, no. February, 2018.

[32] I. Sutskever, J. Martens, G. Dahl, and G. Hinton, "On the importance of initialisation and momentum in deep learning," in Proceedings of the 30th International Conference on Machine Learning, 2013.

[33] T. Dozat, "Incorportating Nesterov Momentum into Adam," 2016.

[34] T. Hastie, R. Tibshirani, and J. J. Friedman, The Elements of Statistical Learning: Data Mining, Inference, and Prediction, 2nd editio. New York: Springer, 2009.

[35] C. M. Bishop, Pattern Recognition and Machine Learning. New York: Springer Verlag New York, 2016.

[36] K. B. Debnath and M. Mourshed, "Forecasting methods in energy planning models," Renew. Sustain. Energy Rev., vol. 88, no. August 2016, pp. 297-325, 2018.

[37] H. R. Khosravani, M. D. M. Castilla, M. Berenguel, A. E. Ruano, and P. M. Ferreira, "A comparison of energy consumption prediction models based on neural networks of a bioclimatic building," Energies, vol. 9, no. 1, pp. 142-155, 2016.

[38] A. Tsanas and A. Xifara, "Accurate quantitative estimation of energy performance of residential 
buildings using statistical machine learning tools," Energy Build., vol. 49, pp. 560-567, 2012.

[39] J. S. Chou and D. K. Bui, "Modeling heating and cooling loads by artificial intelligence for energyefficient building design," Energy Build., vol. 82, no. 2014, pp. 437-446, 2014.

[40] SmartFactoryOWL, "The smart Factory," 2014. [Online]. Available: http://www.smartfactoryowl.de/index.php/en/industry-4-0. [Accessed: 10-Oct-2019].

[41] A. D'Amico, G. Ciulla, D. Panno, and S. Ferrari, "Building energy demand assessment through heating degree days: The importance of a climatic dataset," Appl. Energy2, vol. 242, pp. 12851306, 2019.

[42] CIBSE, "Degree Days for energy management," 2006.

[43] Gazprom, "The Ultimate Energy Efficiency Guide for Manufacturing Businesses," 2017. [Online]. Available: https://www.gazprom-energy.co.uk/blog/the-ultimate-energy-efficiency-guide-formanufacturing-businesses/. [Accessed: 07-Sep-2018]. 